\title{
Inhibition of Host Arginase Activity Against Staphylococcal Bloodstream Infection by Different Metabolites
}

\author{
Rui Pang ${ }^{1}$, Hua Zhou ${ }^{2}$, Yifeng Huang ${ }^{3}$, Yubin Su ${ }^{3 *}$ and Xinhai Chen ${ }^{4,5 *}$ \\ ${ }^{1}$ Guangdong Provincial Key Laboratory of Microbial Safety and Health, State Key Laboratory of Applied Microbiology \\ Southern China, Guangdong Institute of Microbiology, Guangdong Academy of Sciences, Guangzhou, China, ${ }^{2}$ Department \\ of Respiratory and Critical Care Medicine, School of Medicine, The First Affiliated Hospital, Zhejiang University, Hangzhou, \\ China, ${ }^{3}$ Key Laboratory of Functional Protein Research of Guangdong Higher Education Institutes, Department of \\ Biotechnology, College of Life Science and Technology, Jinan University, Guangzhou, China, ${ }^{4}$ Shenzhen International Institute \\ for Biomedical Research, Shenzhen, China, ${ }^{5}$ Department of Microbiology, University of Chicago, Chicago, IL, United States
}

Staphylococcus aureus is a notorious bacterial pathogen that often causes soft tissue and bloodstream infections and invariably garners resistance mechanisms against new antibiotics. Modulation of the host immune response by metabolites is a powerful tool against bacterial infections, but has not yet been used against $S$. aureus infections. In this study, we identified four metabolite biomarkers: L-proline, L-isoleucine, L-leucine, and L-valine (PILV), through a metabolomics study using animal models of S. aureus bloodstream infection. The exogenous administration of each metabolite or of PILV showed anti-infective effects, and a higher protection was achieved with PILV in comparison to individual metabolites. During the staphylococcal infection, the expression of most host arginase and nitric oxide synthase (NOS) isozymes was simultaneously induced in mouse liver, kidney, and blood samples. However, the induction of arginase isozymes was dramatically stronger than that of NOS isozymes. This elevated arginase activity was inhibited by the metabolite biomarkers thus killing S. aureus, and PILV exhibited the strongest inhibition of arginase activity and bacterial inhibition. The suppression of arginase activity also contributed to the metabolite-mediated phagocytic killing of $S$. aureus in mouse and human blood. Our findings demonstrate the metabolite-mediated arginase inhibition as a therapeutic intervention for $S$. aureus infection.

Keywords: metabolite, Staphylococcus aureus, bloodstream infection, arginase, nitric oxide

\section{INTRODUCTION}

Staphylococcus aureus is both, a human commensal and a significant cause of hospital- and community-acquired diseases, including soft tissue infections, pneumonia, osteomyelitis, septic arthritis, bacteremia, endocarditis, and sepsis (1-3). Among the invasive strains isolated from the blood of patients with S. aureus bacteremia, $80 \%$ are genetically indistinguishable from the nasal strains detected at the moment of admission (4). Due to the high incidence of hospital-acquired infections, antibiotics are used for S. aureus decolonization and for prophylaxis of nosocomial disease $(5,6)$. However, the emergence and spread of drug-resistant strains, notably methicillin-resistant $S$. aureus (MRSA), lead to increased therapeutic failure and mortality rates (6), and therefore, new approaches are needed for treating such infections in the clinic. One possible strategy 
would be to enhance the innate immune response of the infected host, thereby restoring its ability to kill the bacterial pathogen in a relatively risk-free manner (7).

S. aureus infection causes several expressive metabolism changes in the host, including oxidative phosphorylation, aerobic glycolysis, and amino acid and fatty acid metabolisms $(8-10)$. These altered pathways have two leading roles: to facilitate bacterial invasion or to benefit the immune response against the infection. The host central carbon metabolism is strongly disturbed by $S$. aureus, which activates autophagy by increasing phosphorylation of AMP-activated protein kinases and extracellular signal-regulated kinases, thereby meeting the staphylococcal invasion (11). Furthermore, the internalization of $S$. aureus destroys the host's arginine metabolism and limits the production of nitric oxide, which serves in the host antibacterial defense, eventually inducing cell death $(12,13)$. In contrast, studies focusing on the cross-talk between metabolic regulation and the immune system reveal an active role of metabolic regulation in the control of pathogenic bacteria. In several bacterial infection models, hosts that survive the infection display distinctive metabolic pathways (14-18). Many of the metabolites of these metabolic pathways are immunoregulators that act via various mechanisms including the activation of PI3K/Akt1, elevated expression of cytokines, and NO production (14-18). To the best of our knowledge, the modulation of host innate immunity by metabolites has not been demonstrated as a valuable strategy against staphylococcal infection thus far.

Here, we used a gas chromatography-mass spectrometry (GCMS) technique to identify metabolites from BALB/c mice infected by three sublethal doses of $S$. aureus strain Newman. The results suggest that four metabolites (L-proline, L-isoleucine, L-leucine, and L-valine) target NO production in order to kill S. aureus and may aid in the development of therapeutic interventions that can improve the outcome of staphylococcal infections.

\section{MATERIALS AND METHODS}

\section{Bacterial Strains, Culture Conditions, and Experimental Animals}

S. aureus strains Newman (ATCC 25904), USA300 (ATCC BAA1717), or MRSA252 (ATCC BAA-1720) were cultured in tryptic soy agar (TSA) at $37^{\circ} \mathrm{C}$. A single colony was grown in tryptic soy broth (TSB) at $37^{\circ} \mathrm{C}$ overnight. The cells were washed and re-suspended in sterile phosphate-buffered saline (PBS). Female BALB/c mice (6 weeks old) were reared in specific pathogen-free (SPF) facilities and fed with dry pellet diets and sterile water. The mice were kept at $20-24^{\circ} \mathrm{C}, 45-65 \%$ relative humidity and a light/dark cycle of $12 / 12 \mathrm{~h}$ in group housings. Each mouse was then intravenously infected with a low $(0.3 \times$ $\left.10^{7}\right)$, moderate $\left(0.7 \times 10^{7}\right)$, or high $\left(1 \times 10^{7}\right)$ concentration (colony-forming units, CFU) of $S$. aureus Newman or with sterile PBS (no infection group) according to previous studies $(19,20)$. Samples of $50 \mu \mathrm{L}$ blood were collected from the orbital vein of each mouse $12 \mathrm{~h}$ post-infection. Animal research was approved by the Institutional Animal Care and Use Committee at the Guangdong Institute of Microbiology (Animal Welfare Assurance Number GT-IACUC201907031).

\section{Metabolomics Analysis}

The metabolite extraction was performed following methods described previously (18). Briefly, $50 \mu \mathrm{L}$ plasma was quenched using $50 \mu \mathrm{L}$ cold methanol and the supernatant was collected by centrifugation at $8,000 \mathrm{rpm}$ for $3 \mathrm{~min}$. Subsequently, Ribitol (5 $\mu \mathrm{L}, 0.1 \mathrm{mg} / \mathrm{mL}$ ) was added as an internal analytical standard, and samples were dried. Sample derivatization and the subsequent data analysis were carried out as described previously $(17,18)$. Samples were oximated and derived, and then analyzed by GC-MS (Trace DSQ II, Thermo Scientific, Waltham, USA). Two technical replicates were conducted for each sample. The metabolomics data was deposited to FigShare (https://doi.org/10. 6084/m9.figshare.12366554).

For data processing, spectral deconvolution and calibration were performed using the automated mass spectral deconvolution \& identification system (AMDIS) and internal standards. Metabolites from the GC-MS spectra were identified by the National Institute of Standards and Technology (NIST) MS search 2.0. The resulting normalized peak intensities formed a single matrix with $\mathrm{Rt}-\mathrm{m} / \mathrm{z}$ pairs for each file in the dataset. The data were median centered and inter-quartile ranges (IQR) were scaled per sample (21). ClustVis was employed to create a principal component analysis (PCA) plot and heatmaps (22). Metabolic pathways were enriched using MetaboAnalyst 4.0 (23).

\section{Effect of Metabolites on S. aureus Infection}

$\mathrm{BALB} / \mathrm{c}$ mice were randomly divided into groups for investigating the effect of L-proline, L-isoleucine, L-valine, L-leucine, or a mixture of all four metabolites (designated as PILV). Hundred microliter of a solution containing each metabolite $\left(0.5 \mathrm{~g} \mathrm{~kg}^{-1}\right)$ or an equal volume of sterile saline solution (no metabolite control) were intraperitoneally injected into each mouse. After $6 \mathrm{~h}$, mice were intravenously challenged by $S$. aureus Newman $\left(1 \times 10^{7} \mathrm{CFU} /\right.$ mouse $)$ and continued receive the metabolites once a day. Body weight was measured daily. All mice were euthanized 15 days post-infection and the kidneys were separated. The bacterial load in each organ was detected by homogenizing, diluting and sampling on TSA. The remaining organ was investigated by histopathology analysis (24). For survival, BLAB/c mice were intravenously inoculated with $100 \mu \mathrm{l}$ of bacterial suspension at a concentration of $2 \times$ $10^{8} \mathrm{CFU} \mathrm{ml}^{-1}$ (USA300) or $2 \times 10^{9} \mathrm{CFU} \mathrm{ml}{ }^{-1}$ (MRSA252). Each metabolite, PILV, BEC [S-(2-boronoethyl)-L-cysteine, an arginase inhibitor at $50 \mathrm{mg} \mathrm{kg}^{-1}$ ], or both a NO inhibitor (LNMMA or L-NAME, $40 \mathrm{mg} \mathrm{kg}^{-1}$ ) and PILV were administrated as mentioned. PILV was administrated with $100 \mu \mathrm{l}$ PBS at a concentration of $0.5 \mathrm{~g} \mathrm{~kg}^{-1}$ of each metabolite. Survival was monitored over 14 days.

\section{Determination of Arginase Activity, NO Release, and Urea}

NO release in serum or tissues was calculated by examining the nitrate and nitrite concentrations with a Total Nitric Oxide Assay kit (Beyotime, Haimen, China). Optical densities at $540 \mathrm{~nm}$ 


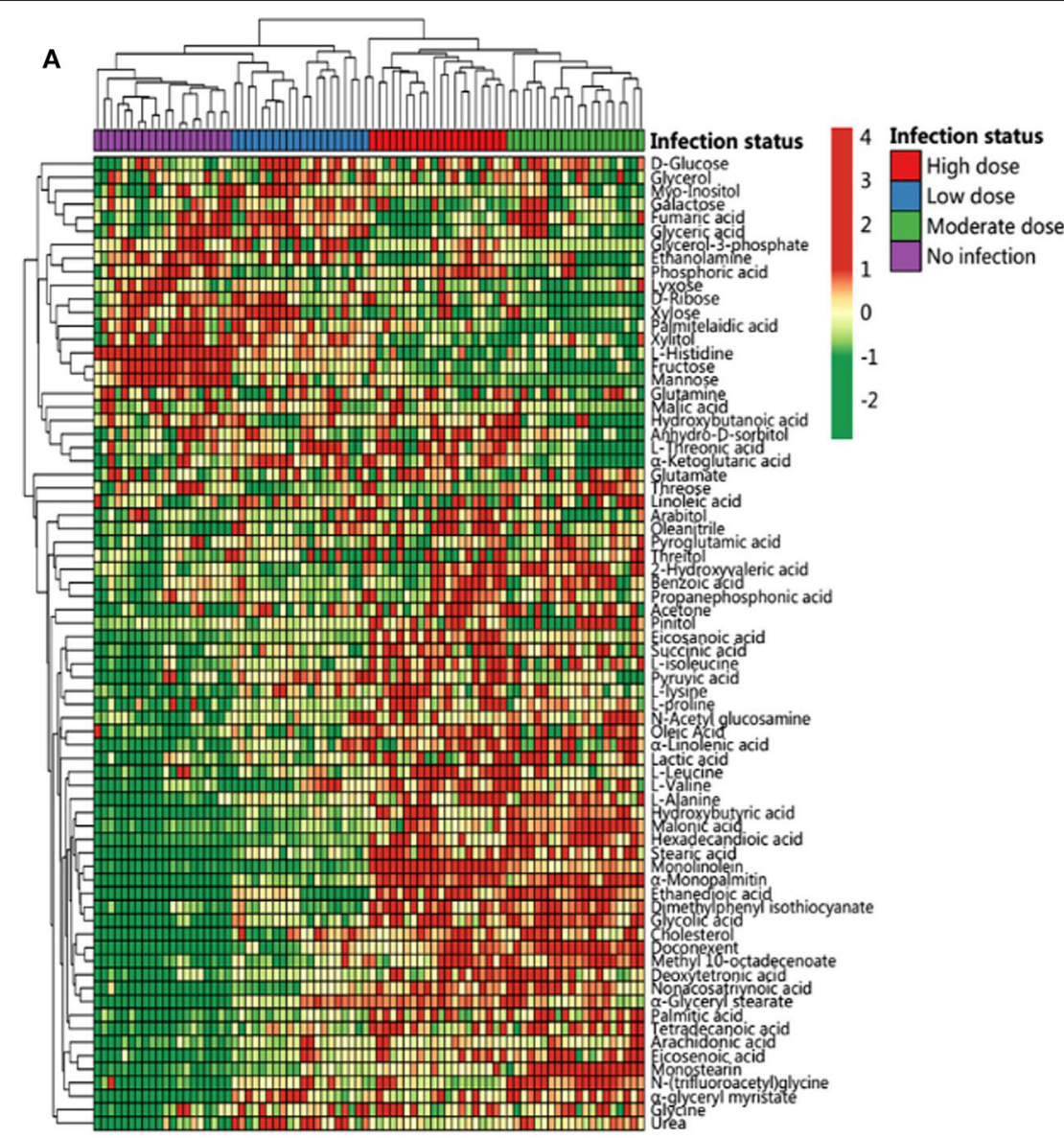

B

S. aureus Newman

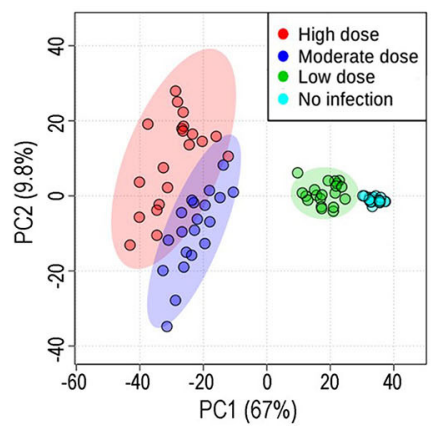

C

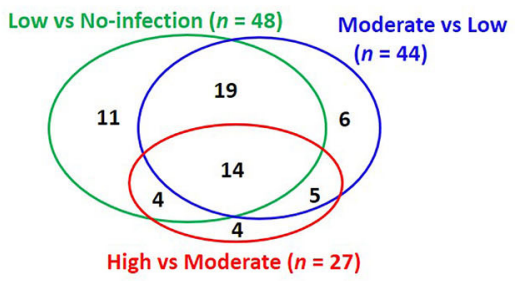

FIGURE 1 | Metabolic profiles of mouse sera injected with different doses of $S$. aureus Newman. (A) The heat map for relative abundances of differential metabolites in low, moderate, and high dose of $S$. aureus Newman strain compared with no-infection control $(n=10$, technical replicate $=2)$. Red and green indicated the increased and decreased of metabolite, respectively (see color scale). (B) Principal component analysis (PCA) of metabolomics data. Each dot showed a technical replicate for one sample. (C) Venn diagram showing the number of significant metabolites between pairwise comparison from no-infection group to high-dose group.

were verified using a microplate reader (Biotek Instruments, Winooski, USA) and NO concentrations were calculated from a standard curve. Urea production in serum was determined using a Urea Colorimetric Assay kit (BioVision, Milpitas, USA). Mouse serum, kidney and liver were collected for the Arginase Activity Assay kit (MAK112, Sigma-Aldrich, San Luis, USA) according to the manufacturer's instructions.

\section{Quantitative Real Time-Polymerase Chain Reaction (qRT-PCR)}

Total RNA was isolated from blood and tissues using TRIzol reagent (Invitrogen, Carlsbad, USA). The cDNA was synthesized using the PrimeScript ${ }^{\mathrm{TM}}$ RT reagent Kit with the genomic DNA Eraser (Takara, Kusatsu, Japan). The mRNA levels of genes Arg1, Arg2, Nos1, Nos2, and Nos3 were detected using qRT-PCR with TB Green ${ }^{\mathrm{TM}}$ Premix Ex Taq ${ }^{\mathrm{TM}}$ II (Takara) in a LightCycler96 system (Roche, Penzberg, Germany). The housekeeping gene coding for $\beta$-Actin (Actb) was used as an endogenous control. All primers are listed in Table S1. After three repeated PCR reactions, the gene expression levels were calculated using the $2^{-\Delta \Delta \mathrm{CT}}$ method (25).

\section{Bacterial Survival in Human and Mouse Blood}

All experiments using blood from human volunteers were approved by the Guangdong Institute of Microbiology's Institutional Review Board (IRB), and informed consent was obtained from all participants. Fresh human blood was collected with heparin. A volume of $0.45 \mathrm{ml}$ of blood was pretreated with $50 \mu \mathrm{l}$ of heat-killed S. aureus Newman $\left(5 \times 10^{5} \mathrm{CFU}\right)$ at $37^{\circ} \mathrm{C}$ for $30 \mathrm{~min}$ and then mixed with $50 \mu \mathrm{l}$ of a live $5 \times 10^{6}$-CFU bacterial suspension in the presence or absence of PILV (10 mM for each metabolite), NO inhibitor (L-NMMA or L-NAME), or both. For mouse blood studies, $100 \mu \mathrm{l}$ of heat-killed S. aureus Newman $\left(5 \times 10^{5} \mathrm{CFU}\right)$ were intravenously injected into BALB/c mice and blood was collected by cardiac puncture after $6 \mathrm{~h}$. A live bacterial suspension $(50 \mu \mathrm{l})$ including $5 \times 10^{5} \mathrm{CFU}$ S. aureus Newman was mixed with $0.45 \mathrm{ml}$ of mouse blood in the presence or absence of PILV (10 mM for each metabolite), NO inhibitor 

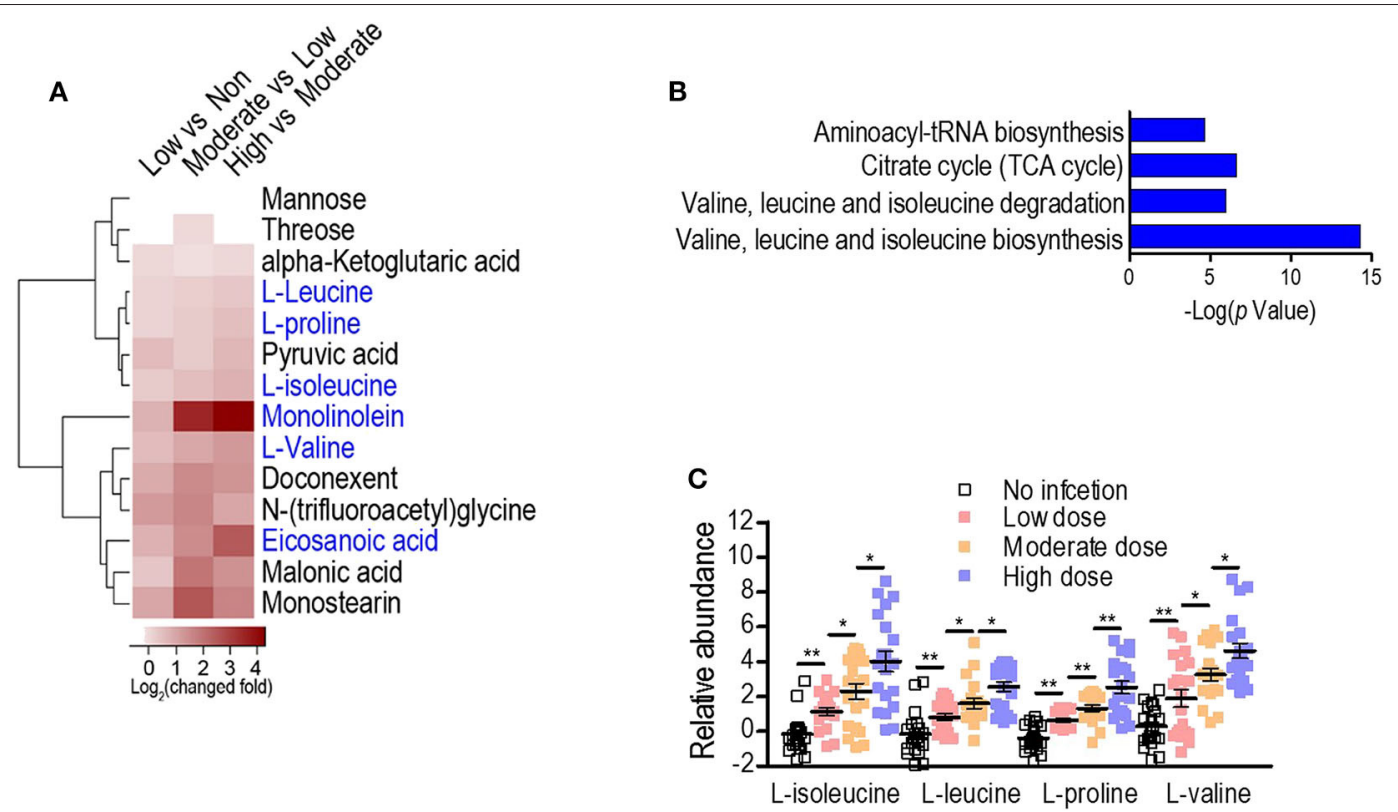

FIGURE 2 | Serum metabolome analysis reveals L-isoleucine, L-leucine, L-proline, and L-valine as potential anti-infection metabolites against S. aureus infection. (A) Heat map representation of unsupervised hierarchical clustering of significant metabolites. (B) Pathway enrichment analysis of the differential metabolites. A horizontal histogram shows the impact of the enriched pathway with $P<0.01$. (C) The abundance of L-isoleucine, L-leucine, L-proline, and L-valine in no infection, Low, Moderate, and High dose groups. Error bars \pm SEM $(n=10$, technical replicate $=2),{ }^{\star} P<0.05$ and ${ }^{\star \star} P<0.01$ (one-way ANOVA).

(L-NMMA or L-NAME), or both. All samples were incubated at $37^{\circ} \mathrm{C}$ with slow rotation for $60 \mathrm{~min}$. Each sample then received $0.55 \mathrm{ml}$ of lysis buffer ( $0.5 \%$ saponin, $200 \mathrm{U}$ streptokinase, 100 $\mu \mathrm{g}$ trypsin, $2 \mu \mathrm{g}$ DNase, $10 \mu \mathrm{g}$ RNase per ml PBS) and was incubated for $10 \mathrm{~min}$ at $37^{\circ} \mathrm{C}$ before plating on TSA $(19,20)$.

\section{Cell Culture and Quantitative Phagocytosis Assay}

The murine macrophage cell line RAW264.7 was cultured in Dulbecco's Modified Eagle Medium (DMEM) supplemented with $10 \%(\mathrm{~V} / \mathrm{V})$ fetal bovine serum (FBS, HyClone, Logan, USA). The human macrophage cell line U937 was grown in Roswell Park Memorial Institute (RPMI) 1640 medium supplemented with $10 \%$ FBS. All cells were grown in a $37^{\circ} \mathrm{C}$ incubator with $5 \% \mathrm{CO}_{2}$. U937-derived macrophages were induced by $160 \mathrm{nM}$ phorbol 12 -myristate 13 -acetate (PMA) at $37^{\circ} \mathrm{C}$ for $48 \mathrm{~h}$. Macrophage phagocytosis was investigated as described previously $(17,18)$. After pretreatment with PILV, NO inhibitor, or both for $6 \mathrm{~h}$, fluorescein (FITC)-conjugated S. aureus was centrifuged onto RAW264.7 or U937-derived macrophages at a multiplicity of infection (MOI) of 100 . After $1 \mathrm{~h}$ of incubation, the macrophages were washed at least four times in cold PBS and then fixed in $4 \%$ paraformaldehyde before harvesting with cold PBS containing $5 \mathrm{mM}$ ethylenediamine tetraacetic acid (EDTA). Finally, cells were subjected to fluorescence-activated cell sorting (FACS) analysis.

\section{Statistical Analysis}

The relative abundances of metabolites in different groups were analyzed with one-way ANOVA (analysis of variance).
Staphylococcal survival in blood, NO and urea levels, and macrophage phagocytosis were analyzed with the two-tailed Student's $t$-test. Bacterial loads and abscess numbers in renal tissues were analyzed with the two-tailed Mann-Whitney test. Statistical significance of survival curves was evaluated by longrank test. All data analysis was performed with GraphPad Prism 8 (GraphPad Software, Inc.), and $P<0.05$ were considered statistically significant.

\section{RESULTS}

\section{GC-MS-Based Metabolomics Identifies Host Metabolites Related to S. aureus Infection}

To exploit anti-infection metabolites, metabolic profiling with different degrees of anti-infection was performed. We hypothesized that different sublethal infection doses would induce different degrees of anti-infection response by the host. Therefore, each group of BALB/c mice $(n=10)$ was intravenously challenged with low, moderate, or high doses of S. aureus Newman or with PBS, and a GC-MS-based approach was used to identify plasma metabolites in challenged mice. All mice survived without signs of toxicity or adverse events. A total of 72 metabolites were detected and displayed as a heat map (Figure 1A). The majority of metabolites suffered abundant changes. Results of PCA were able to distinguish no infection, low dose, moderate dose, and high dose groups (Figure 1B), demonstrating that hosts infected by different sublethal doses displayed differential anti-infection metabolic 
profiles. When comparing no-infection and low-dose groups, low-dose and moderate-dose groups, and moderate-dose and high-dose groups, 48, 44, and 27 differential metabolites were detected, respectively (Figure 1C). Among these, 14 differential metabolites were common to all comparisons. A subset of six metabolites (L-leucine, L-proline, L-isoleucine, monolinolein, Lvaline, and eicosanoic acid) was significantly increased along with the infection dose (Figure 2A). In addition, the 14 differential metabolites shared among all dose comparisons enriched for four pathways: aminoacyl-tRNA biosynthesis; citrate cycle; valine, leucine, and isoleucine degradation; and valine, leucine, and isoleucine biosynthesis $(P<0.05)$ (Figure 2B). Out of the six metabolite biomarkers, L-leucine, L-proline, L-isoleucine, and Lvaline were enriched in the following pathways: aminoacyl-tRNA biosynthesis; valine, leucine, and isoleucine degradation; and valine, leucine, and isoleucine biosynthesis. Therefore, L-leucine, L-proline, L-isoleucine, and L-valine were chosen for further investigation (Figure 2C).

\section{Exogenous Metabolites Display Anti-infective Effect on S. aureus Infection} To examine the anti-infective role of L-leucine, L-proline, Lisoleucine, or L-valine in vivo, cohorts of mice were intravenously infected with $S$. aureus Newman $\left(1 \times 10^{7}\right.$ CFU $)$ and injected with each metabolite $\left(0.5 \mathrm{~g} \mathrm{~kg}^{-1}\right)$ or sterile saline (no metabolite control) daily. When compared to the control group, metabolite-injected mice had significantly reduced weight loss, and staphylococcal load and abscess lesion in kidney tissues (two key disease outcome measures) during the $S$. aureus infection (Figures 3A-C). Each metabolite administration also provided distinct protection against a lethal bloodstream infection with USA300 $\left(2 \times 10^{8} \mathrm{CFU}\right)$ and MRSA252 (2 $\times 10^{9} \mathrm{CFU}$ ) (Figure 3D). There was no difference in body weight recovery, bacterial loads, abscess numbers, and survival among all metabolites (Figures 3A-D). More importantly, the combined administration of all metabolites (PILV) was capable of promoting longer survival (Figure 3D), which was not reached by the administration of a 4-fold concentration of L-proline $\left(2.0 \mathrm{~g} \mathrm{~kg}^{-1}\right)$ (Figure S1); this indicated a synergistic effect of the four metabolites against the staphylococcal infection. Notably, the PILV combination treatment had no influence on the body weight and kidney morphology of mice without $S$. aureus infection (Figure S2). These data suggest that L-leucine, Lproline, L-isoleucine, or L-valine have anti-infective functions during S. aureus infection, and a PILV combination treatment could further improve the outcome of a MRSA infection.

\section{Metabolites Inhibit Arginase Activity to Enhance NO Production in vivo}

Since L-valine can increase NO production in macrophages by inhibiting arginase activity (18), we asked whether L-leucine, L-proline, L-isoleucine, L-valine, or PILV could enhance NO production by blocking the arginase activity in $S$. aureuschallenged mice ( $n=5$ in each group). Firstly, we investigated whether a staphylococcal infection induced both ARG and NOS expressions simultaneously. NO production in serum samples and the expression levels of two ARG (cytoplasmic and mitochondrial arginases, designated as Arg1 and Arg2) and three NOS isozymes (neuronal, inducible, and endothelial NOS, designated as Nos1, Nos2, and Nos3) were measured in tissues and blood upon $S$. aureus infection. Three days after a sublethal infection with $S$. aureus Newman, NO production was enhanced in a dose-dependent manner (Figure 4A). Furthermore, the intravenous infection with $S$. aureus USA300 triggered the expression of all arginase and NOS isozymes and increased NO production and arginase activity in mouse tissues (liver and kidney) and blood (or serum). Nos3 expression was exceptionally unchanged in blood (Figures 4B-D). More interestingly, the $S$. aureus infection induced the expression of ARG isozymes in tissues more strongly than NOS isozymes (Figure 4B). This suggests that both ARG isozymes are predominant regulators of L-arginine, since ARG and NOS compete for L-arginine as an enzyme substrate (26). Cohorts of mice received a daily intraperitoneal injection of each metabolite $\left(0.5\right.$ or $\left.2.0 \mathrm{~g} \mathrm{~kg}^{-1}\right)$ or PILV $\left(0.5 \mathrm{~g} \mathrm{~kg}^{-1}\right.$ of each metabolite) and were infected by $S$. aureus $6 \mathrm{~h}$ after the first injection. On day 3, animals were euthanized, and their blood and tissues (kidney and liver) were collected for measurements of NO production, arginase activity, and urea levels. The mice that received PILV showed the highest levels of NO in sera and tissues and the lowest arginase activities and urea levels in sera, followed by those that received one metabolite or a 4-fold concentration of that metabolite (Figures 4C,D). In the absence of a $S$. aureus infection, 0.5 or $2.0 \mathrm{~g} \mathrm{~kg}^{-1}$ of each metabolite or PILV $\left(0.5 \mathrm{~g} \mathrm{~kg}^{-1}\right.$ of each metabolite) showed no impact on NO production (Figure S3). During the staphylococcal infection, urea content was reduced by single-metabolite treatment and further decreased by PILV treatment (Figure S4A). These data suggest that L-leucine, Lproline, L-isoleucine, or L-valine can strengthen NO production, and PILV combination therapy has an additive effect on NO production through a stronger inhibition of arginase.

\section{Metabolite-Mediated Arginase Inhibition Protects Mice Against S. aureus Infection}

Considering the positive correlation between infective protection and arginase inhibition after PILV treatment, we hypothesized that PILV-induced arginase inhibition could be responsible for a higher protection. We therefore tested this effect using a competitive arginase inhibitor, BEC, which showed no effect on NO production and urea levels under physiological conditions but enhanced NO production (Figure 5A) and decreased urea levels in serum samples of $S$. aureus-infected mice $(n=10$ for each group) (Figure S4B). The survival assay indicated that BEC protected against lethal challenge with MRSA strain USA300 (Figure 5B). These data show that increasing NO production was beneficial in case of a MRSA infection. We then investigated the effects of two NO inhibitors, 1-NMMA, and l-NAME, on PILVinduced NO production, and survival. As expected, the inhibitors significantly suppressed NO production induced by $S$. aureus infection in the absence or presence of PILV (Figure 5C). The mouse survival caused by $S$. aureus infection and enhanced by PILV was reduced by the NO inhibitors (Figure 5D). Together, 
A

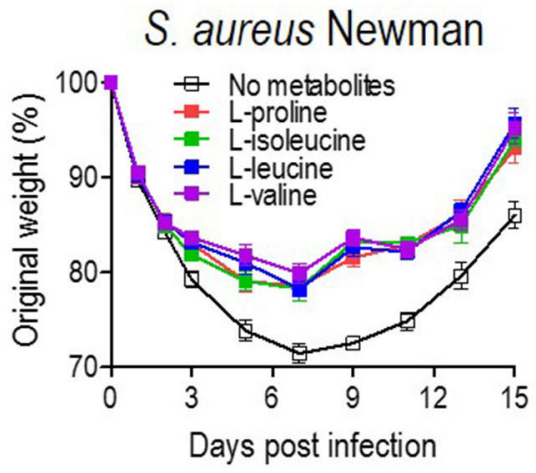

B

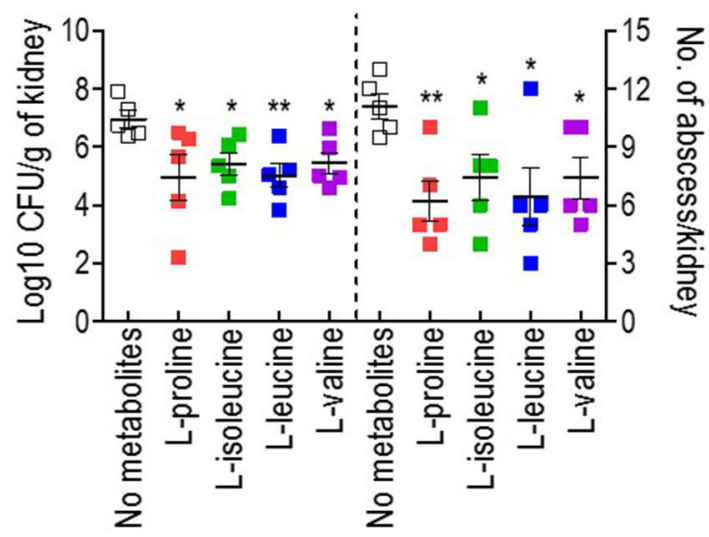

C

$+S$. aureus Newman
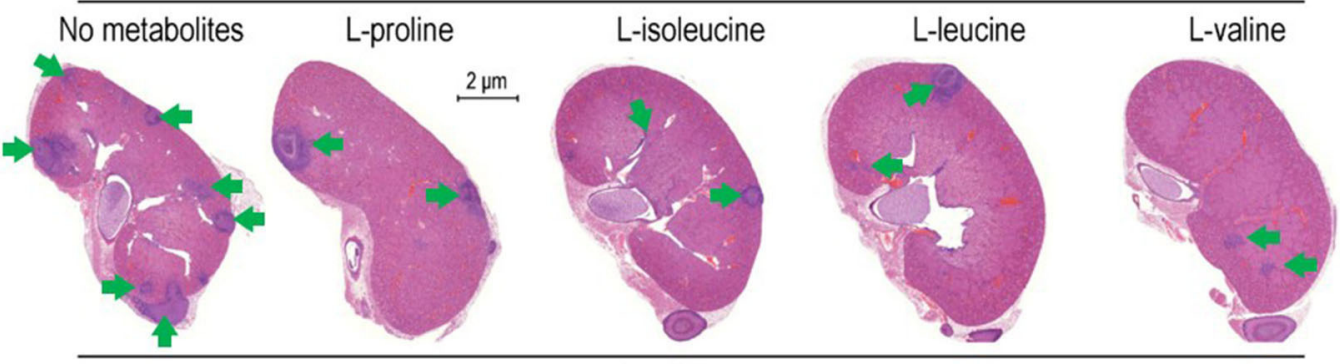

Day 15
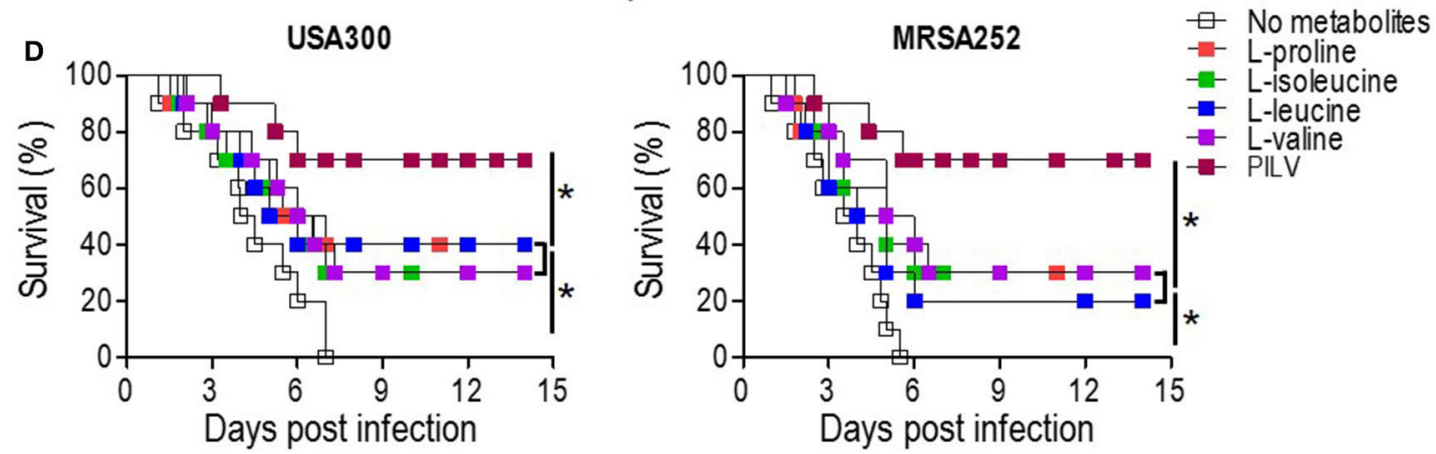

FIGURE 3 | The administration of single metabolite or of a metabolite combination (named PILV) protects mice against S. aureus bloodstream infection. (A-C) Treatment with each metabolite, L-proline, L-isoleucine, L-leucine, or L-valine, rescued body weight loss (A) and reduced renal bacterial loads and abscess numbers $\mathbf{( B , C )}$ in mice infected by S. aureus Newman strain. Weight was recorded daily and reported as \% of initial weight. Fifteen days post-infection, kidneys $(n=5)$ were removed and either ground for enumerating CFU/g tissue or fixed for counting surface abscesses (B). Fixed kidneys were additionally thin sectioned and then stained with hematoxylin and eosin (H\&E) for internal abscesses (C). Green arrows point to internal abscesses in the kidney. (D) Treatment with single metabolites or a metabolite combination (PILV) protected mice (BALB/c, $n=20$ ) against lethal bloodstream infection with S. aureus USA300 and MRSA252. Survival was monitored over 14 days. Data are represented as means \pm SEM. ${ }^{*} P<0.05$ and ${ }^{\star \star} P<0.01$ (two-tailed Mann-Whitney test).

these data indicate that PILV-induced arginase inhibition and subsequent $\mathrm{NO}$ production are able to confer protection against staphylococcal diseases.

\section{Metabolites Increase Phagocytic Killing of $S$. aureus in a NO-Dependent Manner via Arginase Inhibition}

Next, we investigated if PILV had a function in human blood. $S$. aureus opsonophagocytic killing (OPK) was measured in human blood infected with $5 \times 10^{6} \mathrm{CFU}$ Newman for $60 \mathrm{~min}$. Before the measurement, blood was pretreated with heat-killed S. aureus Newman for $30 \mathrm{~min}$ at $37^{\circ} \mathrm{C}$. When added to blood samples, PILV reduced the bacterial load to $75 \%$ (Figure 6A), indicating an anti-infective role of PILV in human blood. Treatment of human blood with a NO inhibitor abolished OPK of Newman in the absence or presence of PILV (Figure 6A). Similar results were found when measuring the OPK of $S$. aureus in mouse blood (Figure 6B). Furthermore, the specific phagocytosis of $S$. aureus Newman was determined in macrophage cell line 

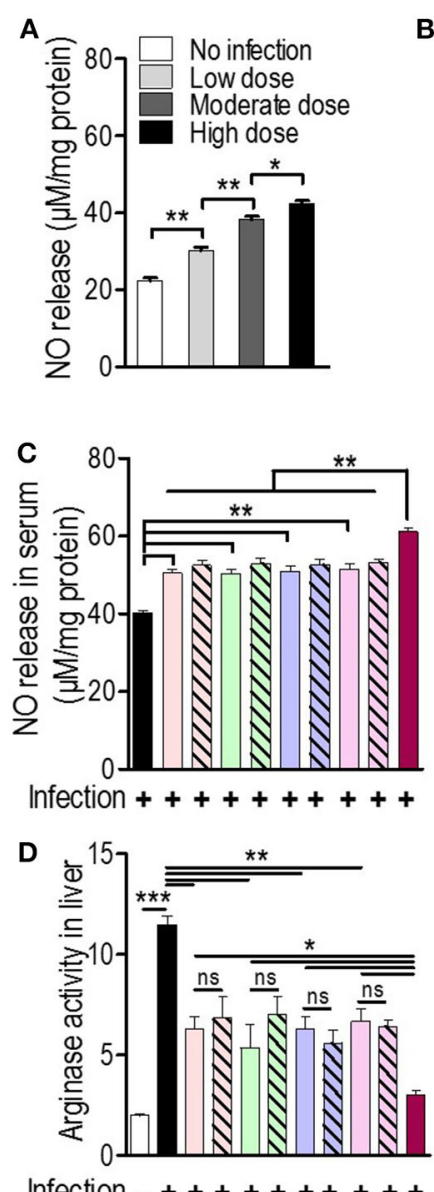

B
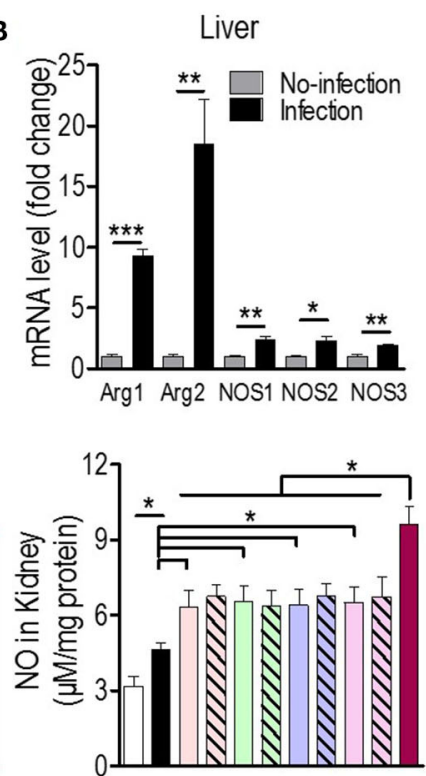

Infection -++++++++++

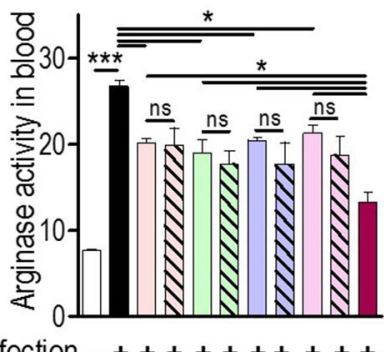

Infection -++++++++++
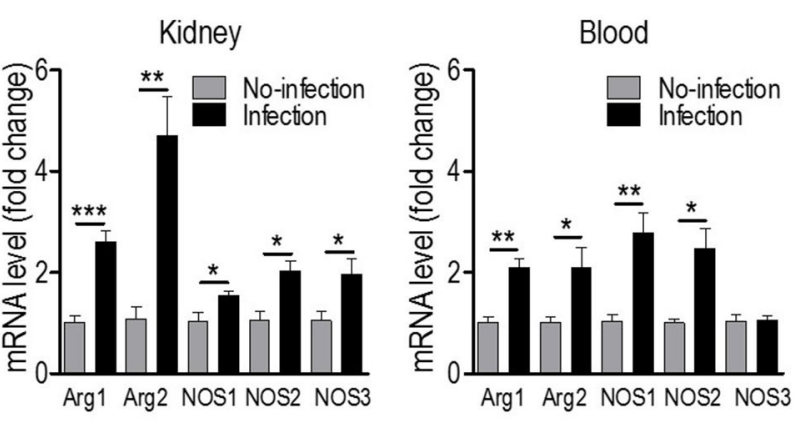

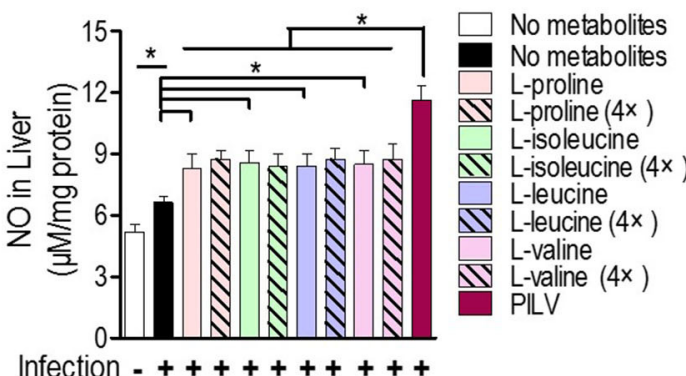

Infection -++++++++++

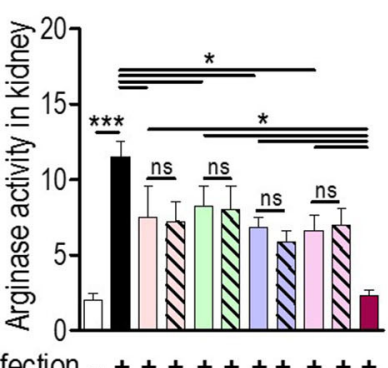

FIGURE 4 | Metabolites promote NO production by arginase inhibition in mice. (A) The NO release under three sublethal doses $\left(0.3 \times 10^{7}, 0.7 \times 10^{7}\right.$, and $1 \times 10^{7}$ $\mathrm{CFU} /$ mouse) of $S$. aureus Newman infection ( $n=5$ for each dose group). Three days post-infection, mouse serum was collected and subjected to measurement of NO release. (B) The mRNA levels of two arginase (ARG) and three NO synthase (NOS) isoforms in mouse liver, kidney, and blood under a sublethal staphylococcal infection. S. aureus USA300 $\left(5 \times 10^{6} \mathrm{CFU} /\right.$ mouse) was used to infect BALB/c mice $(n=5)$. Three days after the infection, the mouse liver, kidney, and blood were collected and the transcriptional expression of ARG and NOS was measured. Arg1 and Arg2 are cytoplasmic and mitochondrial arginases, respectively. Nos 1, Nos2, and Nos3 are neuronal, inducible, and endothelial NOS, respectively. The NO production (C) and arginase activity (D) in mouse blood and tissues treated with single metabolite or PILV under staphylococcal infection. Data are represented as means \pm SEM. ${ }^{\star} P<0.05,{ }^{\star \star} P<0.01$ and ${ }^{\star \star \star} P<0.001$ (two-tailed Student's $t$-test).

RAW264.7 and differentiated U937 cells. As anticipated, the NO inhibitor or cytochalasin D completely depleted the PILVenhanced phagocytosis of $S$. aureus in either human or mouse macrophages (Figures 6C,D). Altogether, these data demonstrate that PILV promotes the phagocytic killing of $S$. aureus in a NO-dependent manner via arginase inhibition.

\section{DISCUSSION}

$\mathrm{NO}$ is a versatile effector that plays a central role in both, antimicrobial activity and immunomodulation. During an infection, the highly cytotoxic NO levels produced by innate immune cells limit pathogen growth (27), and although S. aureus has several genes for efficient NO detoxification, NO production is still critical in host resistance to the staphylococcal disease $(28,29)$. NO targets the Agr quorum sensing system to disrupt cell-to-cell communication in $S$. aureus (30) and acts as a signaling messenger to promote immune cell activity $(27,31)$. Inhibition of NOS in macrophages or neutrophils significantly blocks phagocytosis and intracellular killing, and increases survival of S. aureus $(18,32-34)$. These observations resulted in the development of NO delivery systems that harness the antimicrobial properties of this short-lived gas $(27,35)$. In the present study, we found that the administration of a combination of four metabolites during $S$. aureus infection enhanced endogenous NO production.

Metabolomics is an advanced technology that examines metabolic processes, identifying biomarkers and unraveling mechanisms (36). The analysis of crucial metabolites in samples with different statuses has virtually become a significant part of improving the diagnosis, prognosis, and therapy of diseases (37). Various metabolites, including glucose, malate, $\mathrm{N}$-acetylglucosamine, myo-inositol, linoleic acid, L-proline, L-valine, and L-leucine, have shown extended protection against 

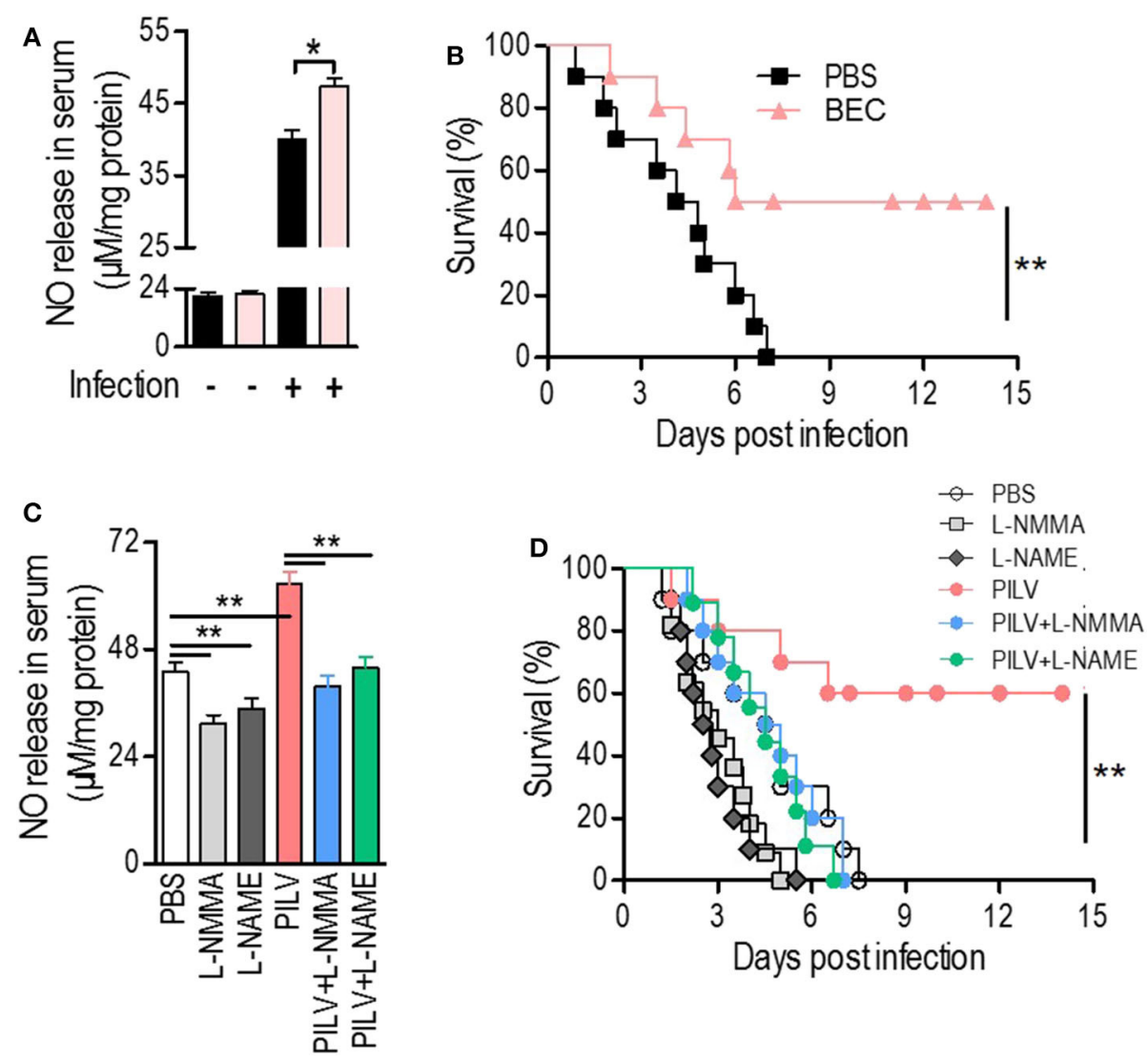

FIGURE 5 | Metabolite-induced NO confers protection against staphylococcal infection. (A,B) The impact of a competitive arginase inhibitor, BEC (S-(2-boronoethyl)-L-cysteine), on NO release in serum (A) and survival (B) of mice under a lethal bloodstream infection with S. aureus USA300. (C,D) The impact of NO inhibitors, L-NMMA, and L-NAME, on NO release in serum (C) and survival (D) of mice under a lethal bloodstream infection with S. aureus USA300. Six hours after the injection of BEC (50 mg kg $\left.{ }^{-1}\right)$, L-NMMA ( $\left.40 \mathrm{mg} \mathrm{kg}^{-1}\right)$, L-NAME $\left(40 \mathrm{mg} \mathrm{kg}^{-1}\right)$, PILV, PILV plus L-NMMA (40 mg kg $\left.{ }^{-1}\right)$, or PILV plus L-NAME (40 mg kg $\left.{ }^{-1}\right)$, BALB/c mice $(n=30)$ were lethally challenged by $S$. aureus USA300 and then divided into two subgroups. One subgroup $(n=10)$ was used for NO measurement and another $(n=20)$ was used for observing survival. Five of the survival mice were euthanized 3 days after the infection to measure NO and urea production in serum. Survival was monitored over 14 days. Data are represented as means \pm SEM. ${ }^{\star} P<0.05$ and ${ }^{\star \star} P<0.01$ (two-tailed Student's $t$-test).

bacterial infections $(14-18,38)$. However, the mechanisms underlying their anti-infection properties are currently minimally understood. Studies showed an inhibitory effect of L-proline, L-isoleucine, L-leucine, and L-valine on arginase activities; this inhibition is relatively specific as other amino acids, such as glycine and L-glutamine, do not influence arginase activities (39-41). Arginase inhibition by L-valine increases NO production in endothelial cells and macrophages during lipopolysaccharide (LPS) treatment (18, 42). Arginase elimination in macrophages favors host survival in Toxoplasma gondii infections and reduces bacterial loads in lung infections with Mycobacterium tuberculosis (43). NOS and arginase are co-induced during staphylococcal infection but the enzymatic role of arginase is distinctly more robust than that of NOS. The induced arginase competes with NOS for L-arginine utilization, which limits the NO-mediated anti-infection effect (26). Interestingly, L-proline, L-isoleucine, L-leucine, and L-valine boosted NO production via arginase inhibition in $S$. aureusinfected hosts. More importantly, the combined administration of all four metabolites provided stronger arginase inhibition and protection against $S$. aureus infection. This happened largely through a mechanism where in the absence of arginase activity, L-arginine is mostly consumed by NOS to produce NO.

There are two isoforms of arginase, arginase I and arginase II, which are located on cytosol and mitochondria, respectively. Branched-chain amino acids (BCAAs) cause significant inhibition of arginase I and only a minor effect on arginase II, while L-proline displays a stronger inhibition of arginase II in comparison to arginase I (39). This possibly explains the stronger arginase inhibition caused by the combination of L-proline and BCAAs but not by a 4 -fold concentration of each metabolite, although further investigation is still required on this matter. Additionally, S. aureus carries its own arginase gene, which might behave like its host counterpart reducing the amount of L-arginine available for $\operatorname{NOS}(12,44,45)$. The inhibitory effect of L-proline and BCAAs on staphylococcal arginase needs to be determined in the future. 

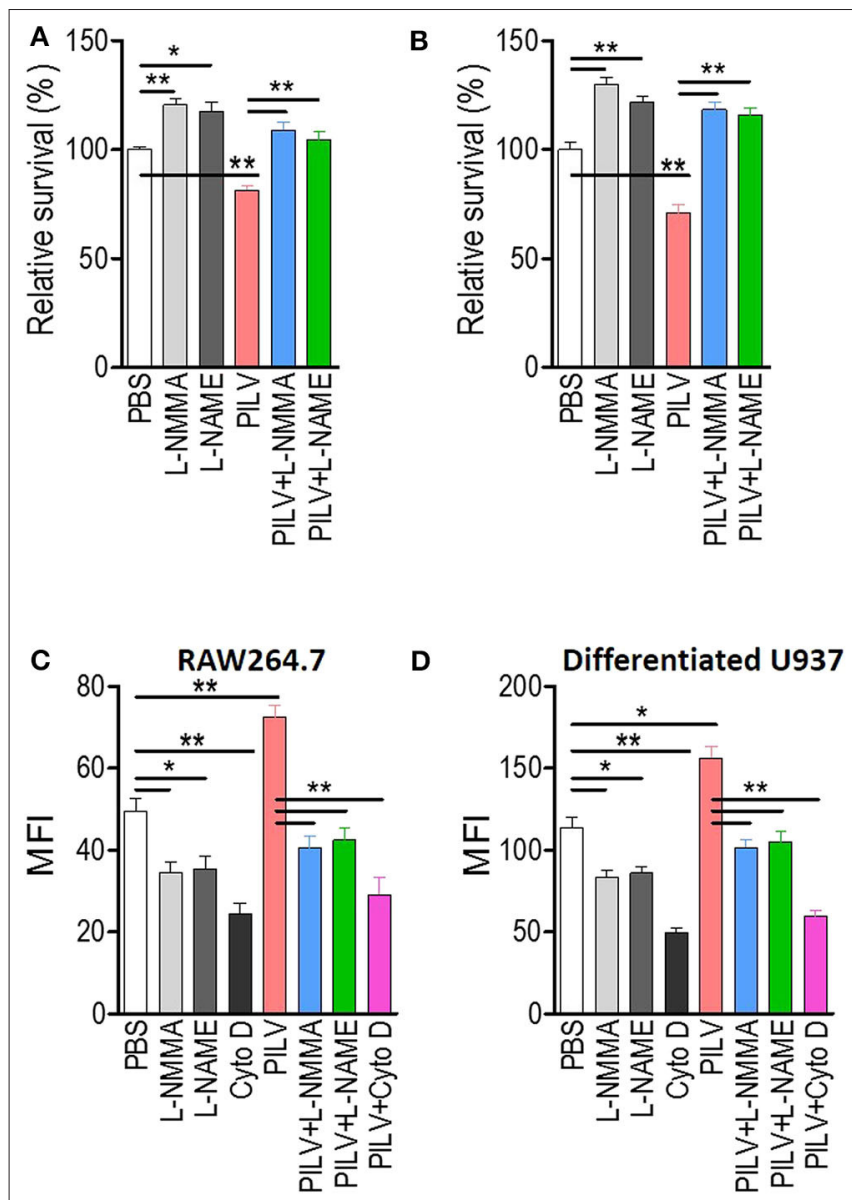

FIGURE 6 | PILV enhances opsonophagocytic killing (OPK) in a NO-dependent manner. (A,B) Relative survival about OPK of $S$. aureus in human (A) and mouse blood (B) without or with PILV. Human and mouse anticoagulated blood treated with heat-killed S. aureus Newman was incubated with live S. aureus Newman $\left(2.5 \times 10^{6} \mathrm{CFU} / \mathrm{ml}\right.$ blood for the human assay, $2.5 \times 10^{5} \mathrm{CFU} / \mathrm{ml}$ blood for the mouse assay) in the presence of PBS, NO inhibitors, PILV, or PILV plus NO inhibitors for $60 \mathrm{~min}$, and survival was measured $(n=5)$. (C,D) Determination the phagocytosis of FITC-conjugated S. aureus Newman in RAW 264.7 (C) and U937-derived macrophages (D) without or with PILV. RAW264.7 or U937-derived macrophages were pretreated with PBS, NO inhibitors, PILV, or PILV plus NO inhibitors in a serum-starved medium for $6 \mathrm{~h}$ and then were co-incubated with FITC-conjugated $S$. aureus for one more hour. Bacterial uptake was measured by flow cytometry. Data are represented as means \pm SEM. ${ }^{\star} P<0.05$ and ${ }^{\star \star} P$ $<0.01$ (two-tailed Student's $t$-test).

The fact that the host employs L-proline and BCAAs as anti-infection metabolites against $S$. aureus bloodstream infection is surprising because $S$. aureus presents amino acid auxotrophies in minimal media regarding L-proline, L-valine, and L-leucine, although its genome contains the gene sets for the biosynthesis of these amino acids (46, 47). However, our results showed an elevation in these amino acids in the plasma of infected animals. Instead of enhancing S. aureus growth, exogenous supplementation of L-proline and BCAAs facilitated the phagocyte-mediated OPK of $S$. aureus and the elimination of staphylococci by the host. Consistently,
S. aureus infection reduces the transcriptional level of BCAA transaminase 2, which mainly contributes to BCAA degradation (48), suggesting a possible lower degradation rate of BCAAs in infected mice. TLR2/TLR6 agonists stimulate a significant increase in L-valine and L-isoleucine in mice (8), revealing that $S$. aureus-derived lipoteichoic acid and peptidoglycan could be the inducer in infected animals. The qRT-PCR data showed that $S$. aureus infection has no impact on TLR2/TLR6 expressions but exogenous L-isoleucine, L-valine, or PILV significantly reduces the level of TLR2 in blood samples (Figure S5). The downregulated expression of TLR2 would cause a weaker immune response from lipoteichoic acid and peptidoglycan stimulations. Thus, in the presence of exogenous L-isoleucine, L-valine, or PILV, the level of proline and BCAAs may not be enhanced by $S$. aureus infection if the TLR2/TLR6-dependent mechanism has been demonstrated. This also seems logical since exogenous addition has been able to meet the demand for boosting NO to kill $S$. aureus, and those biomarkers no longer need to be increased through TLR2/TLR6-dependent mechanism. The reduction of TLR2 expression by L-isoleucine, L-valine, or PILV may have another effect. The inflammation is a double-edged sword; too little is not enough to control infection, and too high would have damage to the body. Thus we presume that the exogenous L-isoleucine, L-valine, or PILV inhibits the inflammation in a TLR2 dependent manner while still boosting NO to kill $S$. aureus. The specific mechanism for this phenomenon remains to be determined in future studies for a better understanding of the metabolite-mediated innate immunity against $S$. aureus infection. Besides the innate immunity, the lymphocyte responserelated adaptive immunity also can be potentially regulated by L-proline and BCAAs to enhance its anti-infectivity. BCAA not only increases fuel sources for $\mathrm{CD}^{+}$and $\mathrm{CD}^{+} \mathrm{T}$ cells and the secretion of intestinal immunoglobulins, but also regulates the development of Treg (regulatory T) cells through mTORC1 signaling $(49,50)$. The proliferation of activated $\mathrm{T}$ cell is rely on the uptake of L-proline (51). The role of BCAA and Lproline on adaptive immunity against $S$. aureus infection should be evaluated in future studies.

\section{DATA AVAILABILITY STATEMENT}

All datasets generated for in this study are included in the article/Supplementary Material.

\section{ETHICS STATEMENT}

The studies involving human participants were reviewed and approved by Institutional Ethics Committee at the Guangdong Institute of Microbiology. The patients/participants provided their written informed consent to participate in this study. The animal study was reviewed and approved by Institutional Animal Care and Use Committee at the Guangdong Institute of Microbiology (Animal Welfare Assurance Number GT-IACUC201907031). 


\section{AUTHOR CONTRIBUTIONS}

$\mathrm{XC}, \mathrm{RP}$, and YS developed methods and conceptualized ideas. $\mathrm{RP}, \mathrm{YS}, \mathrm{HZ}$, and XC designed experiments. XC, RP, YS, and YH performed experiments. XC and RP analyzed data. XC, YS, and $\mathrm{RP}$ wrote the manuscript. All authors contributed to the article and approved the submitted version.

\section{FUNDING}

This work was supported by the National Natural Science Foundation of China grant 31901796, the Natural Science Foundation of Guangdong Province grant 2019A1515012211, the GDIM Young Elite Talents Project grant GDIMYET20170104, the Shenzhen Science and Technology Innovation Commission

\section{REFERENCES}

1. Wertheim HF, Melles DC, Vos MC, van Leeuwen W, van Belkum A, Verbrugh $\mathrm{HA}$, et al. The role of nasal carriage in Staphylococcus aureus infections. Lancet Infect Dis. (2005) 5:751-62. doi: 10.1016/S1473-3099(05)70295-4

2. Lebon A, Labout JA, Verbrugh HA, Jaddoe VW, Hofman A, van Wamel $\mathrm{W}$, et al. Dynamics and determinants of Staphylococcus aureus carriage in infancy: the generation R study. J Clin Microbiol. (2008) 46:351721. doi: 10.1128/JCM.00641-08

3. Verhoeven PO, Gagnaire J, Botelho-Nevers E, Grattard F, Carricajo A, Lucht F, et al. Detection and clinical relevance of Staphylococcus aureus nasal carriage: an update. Expert Rev Anti Infect Ther. (2014) 12:7589. doi: 10.1586/14787210.2014.859985

4. Wertheim HF, Vos MC, Ott A, van Belkum A, Voss A, Kluytmans JA, et al. Risk and outcome of nosocomial Staphylococcus aureus bacteraemia in nasal carriers versus non-carriers. Lancet. (2004) 364:7035. doi: 10.1016/S0140-6736(04)16897-9

5. van Rijen M, Bonten M, Wenzel R, Kluytmans J. Mupirocin ointment for preventing Staphylococcus aureus infections in nasal carriers. Cochrane Database Syst Rev. (2008) CD006216. doi: 10.1002/14651858.CD006216.pub2

6. Simor AE. Staphylococcal decolonisation: an effective strategy for prevention of infection? Lancet Infect Dis. (2011) 11:95262. doi: 10.1016/S1473-3099(11)70281-X

7. Hancock RE, Nijnik A, Philpott DJ. Modulating immunity as a therapy for bacterial infections. Nat Rev Microbiol. (2012) 10:243-54. doi: 10.1038/nrmicro2745

8. Hoerr V, Zbytnuik L, Leger C, Tam PPC, Kubes P, Vogel, et al. Gramnegative and gram-positive bacterial infections give rise to a different metabolic response in a mouse model. J Proteome Res. (2012) 11:323145. doi: 10.1021/pr201274r

9. Holtfreter S, Kolata J, Stentzel S, Bauerfeind S, Schmidt F, Sundaramoorthy $\mathrm{N}$, et al. Omics approaches for the study of adaptive immunity to Staphylococcus aureus and the selection of vaccine candidates. Proteomes. (2016) 4:11. doi: 10.3390/proteomes4010011

10. Ambroggio L, Florin TA, Shah SS, Ruddy R, Yeomans L, Trexel H, et al. Emerging biomarkers of illness severity: urinary metabolites associated with sepsis and necrotizing methicillin-resistant Staphylococcus aureus pneumonia. Pharmacotherapy. (2017) 37:1033-42. doi: 10.1002/phar.1973

11. Bravo-Santano N, Ellis JK, Mateos LM, Calle Y, Keun HC, Behrends $\mathrm{V}$, et al. Intracellular Staphylococcus aureus modulates host central carbon metabolism to activate autophagy. mSphere. (2018) 3:e0037418. doi: $10.1128 / \mathrm{mSphere} .00374-18$

12. Medina LMP, Becker AK, Michalik S, Yedavally H, Raineri EJM, Hildebrandt $\mathrm{P}$, et al. Metabolic cross-talk between human bronchial epithelial cells and internalized Staphylococcus aureus as a driver for infection. Mol Cell Proteomics. (2019) 18:892-908. doi: 10.1074/mcp.RA118.001138
Project grant JCYJ20170815113109175, and the Dapeng Project grant KY20170202.

\section{ACKNOWLEDGMENTS}

We thank W. Yu and J. Madsen (University of South Florida) for comments and discussion. This manuscript has been released as a pre-print at bioRxiv (52).

\section{SUPPLEMENTARY MATERIAL}

The Supplementary Material for this article can be found online at: https://www.frontiersin.org/articles/10.3389/fimmu. 2020.01639/full\#supplementary-material
13. Xiong L, Teng J, Botelho M, Lo RC, Lau SK, Woo PC. Arginine metabolism in bacterial pathogenesis and cancer therapy. Int J Mol Sci. (2016) 17:363. doi: 10.3390/ijms17030363

14. Du CC Yang MJ, Li MY, Yang J, Peng B, Li H, et al. Metabolic mechanism for L-Leucine-induced metabolome to eliminate Streptococcus iniae. J Proteome Res. (2017) 16:1880-9. doi: 10.1021/acs.jproteome.6b00944

15. Yang MJ, Cheng ZX, Jiang M, Zeng ZH, Peng B, Peng XX, et al. Boosted TCA cycle enhances survival of zebrafish to Vibrio alginolyticus infection. Virulence. (2018) 9:634-44. doi: 10.1080/21505594.2017.1423188

16. Ma YM, Yang MJ, Wang S, Li H, Peng XX. Liver functional metabolomics discloses an action of l-leucine against Streptococcus iniae infection in tilapias. Fish Shellfish Immunol. (2015) 45:414-21. doi: 10.1016/j.fsi.2015.04.037

17. Chen XH, Zhang BW, Li H, Peng XX. Myo-inositol improves the host's ability to eliminate balofloxacin-resistant Escherichia coli. Sci Rep. (2015) 5:10720. doi: 10.1038/srep 10720

18. Chen XH, Liu R, Peng B, Li D, Cheng ZX, Zhu JX, et al. Exogenous L-valine promotes phagocytosis to kill multidrug-resistant bacterial pathogens. Front Immunol. (2017) 8:207. doi: 10.3389/fimmu.2017.00207

19. Chen X, Sun Y, Missiakas D, Schneewind O. Staphylococcus aureus decolonization of mice with monoclonal antibody neutralizing protein A. $J$ Infect Dis. (2018) 219:884-8. doi: 10.1093/infdis/jiy597

20. Thomer L, Emolo C, Thammavongsa V, Kim HK, McAdow ME, Yu W, et al. Antibodies against a secreted product of Staphylococcus aureus trigger phagocytic killing. J Exp Med. (2016) 213:293-301. doi: 10.1084/jem.20150074

21. Liu SR, Peng XX, Li H. Metabolic mechanism of ceftazidime resistance in Vibrio alginolyticus. Infect Drug Resist. (2019) 12:417-29. doi: 10.2147/IDR.S179639

22. Metsalu T, Vilo J. ClustVis: a web tool for visualizing clustering of multivariate data using principal component analysis and heatmap. Nucleic Acids Res. (2015) 43:W566-70. doi: 10.1093/nar/gkv468

23. Chong J, Soufan O, Li C, Caraus I, Li S, Bourque G, et al. MetaboAnalyst 4.0: towards more transparent and integrative metabolomics analysis. Nucleic Acids Res. (2018) 46:W486-94. doi: 10.1093/nar/gky310

24. Kim HK, Falugi F, Thomer L, Missiakas DM, Schneewind O. Protein A suppresses immune responses during Staphylococcus aureus bloodstream infection in guinea pigs. MBio. (2015) 6:e02369-14. doi: 10.1128/mBio.02369-14

25. Livak KJ, Schmittgen TD. Analysis of relative gene expression data using real-time quantitative PCR and the 2(-Delta C(T)) method. Methods. (2001) 25:402-8. doi: 10.1006/meth.2001.1262

26. Boucher JL, Moali C, Tenu JP. Nitric oxide biosynthesis, nitric oxide synthase inhibitors and arginase competition for L-arginine utilization. Cell Mol Life Sci. (1999) 55:1015-28. doi: 10.1007/s000180050352

27. Schairer DO, Chouake JS, Nosanchuk JD, Friedman AJ. The potential of nitric oxide releasing therapies as antimicrobial agents. Virulence. (2012) 3:271-9. doi: 10.4161/viru.20328 
28. Kinkel TL, Roux CM, Dunman PM, Fang FC. The Staphylococcus aureus SrrAB two-component system promotes resistance to nitrosative stress and hypoxia. MBio. (2013) 4:e00696-13. doi: 10.1128/mBio.00696-13

29. Richardson AR, Libby SJ, Fang FC. A nitric oxide-inducible lactate dehydrogenase enables Staphylococcus aureus to resist innate immunity. Science. (2008) 319:1672-6. doi: 10.1126/science.1155207

30. Urbano R, Karlinsey JE, Libby SJ, Doulias PT, Ischiropoulos H, Warheit-Niemi $\mathrm{HI}$, et al. Host nitric oxide disrupts microbial cell-to-cell communication to inhibit staphylococcal virulence. Cell host Microbe. (2018) 23:594606.e7. doi: 10.1016/j.chom.2018.04.001

31. Tripathi P, Tripathi P, Kashyap L, Singh V. The role of nitric oxide in inflammatory reactions. FEMS Immunol Med Microbiol. (2007) 51:44352. doi: 10.1111/j.1574-695X.2007.00329.x

32. Li C, Li H, Jiang Z, Zhang T, Wang Y, Li Z, et al. Interleukin-33 increases antibacterial defense by activation of inducible nitric oxide synthase in skin. PLoS Pathog. (2014) 10:e1003918. doi: 10.1371/journal.ppat.1003918

33. Moffat FL Jr, Han T, Li ZM, Peck MD, Jy W, Ahn YS, et al. Supplemental Larginine $\mathrm{HCI}$ augments bacterial phagocytosis in human polymorphonuclear leukocytes. J Cell Physiol. (1996) 168:26-33. doi: 10.1002/(SICI)10974652(199607)168:1<26::AID-JCP4>3.0.CO;2-A

34. Sakiniene E, Bremell T, Tarkowski A. Inhibition of nitric oxide synthase (NOS) aggravates Staphylococcus aureus septicaemia and septic arthritis. Clin Exp Immunol. (1997) 110:370-7. doi: 10.1046/j.1365-2249.1997.4431456.x

35. Park D, Kim J, Lee YM, Park J, Kim WJ. Polydopamine hollow nanoparticle functionalized with $\mathrm{n}$-diazeniumdiolates as a nitric oxide delivery carrier for antibacterial therapy. Adv Healthc Mater. (2016) 5:201924. doi: 10.1002/adhm.201600150

36. Peng B, Li H, Peng XX. Functional metabolomics: from biomarker discovery to metabolome reprogramming. Protein Cell. (2015) 6:62837. doi: 10.1007/s13238-015-0185-x

37. Cheng ZX, Ma YM, Li H, Peng XX. N-acetylglucosamine enhances survival ability of tilapias infected by Streptococcus iniae. Fish Shellfish Immunol. (2014) 40:524-30. doi: 10.1016/j.fsi.2014.08.008

38. Zhao $\mathrm{X}, \mathrm{Wu} \mathrm{C}$, Peng $\mathrm{X}$, Li $\mathrm{H}$. Interferon- $\alpha 2 \mathrm{~b}$ against microbes through promoting biosynthesis of unsaturated fatty acids. J Proteome Res. (2014) 13:4155-63. doi: 10.1021/pr500592x

39. Carvajal N, Cederbaum SD. Kinetics of inhibition of rat liver and kidney arginases by proline and branched-chain amino acids. Biochim Biophys Acta. (1986) 870:181-4. doi: 10.1016/0167-4838(86)90 219-0

40. Pietkiewicz J, Bryła J. Comparison of influence of 2-oxoglutarate and glutamate on arginase activities in liver and kidney-cortex of rabbit, Oryctolagus cuniculus. Comp Biochem Physiol B Biochem Mol Biol. (1996) 115:393-8. doi: 10.1016/S0305-0491(96)00 154-X

41. Rao KK, Reddy SRR, Swami KS. The inhibition of sheep liver arginase by some L-amino-acids. Int $J$ Biochem. (1973) 4:62-70. doi: 10.1016/0020-711X(73)90021-9
42. Chicoine LG, Paffett ML, Young TL, Nelin LD. Arginase inhibition increases nitric oxide production in bovine pulmonary arterial endothelial cells. Am J Physiol Lung Cell Mol Physiol. (2004) 287:L60-8. doi: 10.1152/ajplung.00194.2003

43. El Kasmi KC, Qualls JE, Pesce JT, Smith AM, Thompson RW, HenaoTamayo M, et al. Toll-like receptor-induced arginase 1 in macrophages thwarts effective immunity against intracellular pathogens. Nat Immunol. (2008) 9:1399-406. doi: 10.1038/ni.1671

44. Nuxoll AS, Halouska SM, Sadykov MR, Hanke ML, Bayles KW, Kielian T, et al. CcpA regulates arginine biosynthesis in Staphylococcus aureus through repression of proline catabolism. PLoS Pathog. (2012) 8:e1003033. doi: 10.1371/journal.ppat.1003033

45. Lahiri A, Das P, Chakravortty D. New tricks new ways: exploitation of a multifunctional enzyme arginase by pathogens. Virulence. (2010) 1:56365. doi: 10.4161/viru.1.6.13623

46. Emmett M, Kloos WE. Amino acid requirements of staphylococci isolated from human skin. Can J Microbiol. (1975) 21:729-33. doi: 10.1139/m75-107

47. Kaiser JC, King AN, Grigg JC, Sheldon JR, Edgell DR, Murphy MEP, et al. Repression of branched-chain amino acid synthesis in Staphylococcus aureus is mediated by isoleucine via CodY, and by a leucine-rich attenuator peptide. PLoS Genet. (2018) 14:e1007159. doi: 10.1371/journal.pgen.1007159

48. Koziel J, Maciag-Gudowska A, Mikolajczyk T, Bzowska M, Sturdevant DE, Whitney AR, et al. Phagocytosis of Staphylococcus aureus by macrophages exerts cytoprotective effects manifested by the upregulation of antiapoptotic factors. PLoS ONE. (2009) 4:e5210. doi: 10.1371/journal.pone.0005210

49. Nie C, He T, Zhang W, Zhang G, Ma X. Branched chain amino acids: beyond nutrition metabolism. Int J Mol Sci. (2018) 19:954. doi: 10.3390/ijms19040954

50. MacIver NJ, Michalek RD, Rathmell JC. Metabolic regulation of T lymphocytes. Annu Rev Immunol. (2013) 31:25983. doi: 10.1146/annurev-immunol-032712-095956

51. Chu TLH, Guan Q, Nguan CYC, Du C. Halofuginone suppresses T cell proliferation by blocking proline uptake and inducing cell apoptosis. Int Immunopharmacol. (2013) 16:414-23. doi: 10.1016/j.intimp.2013.04.031

52. Pang R, Su Y, Zhou H, Chen X. Metabolites alleviate staphylococcal bloodstream infection in a NO-dependent manner via arginase inhibition. bioRxiv [Preprint]. (2020). doi: 10.1101/2020.03.02.974345

Conflict of Interest: The authors declare that the research was conducted in the absence of any commercial or financial relationships that could be construed as a potential conflict of interest.

Copyright (c) 2020 Pang, Zhou, Huang, Su and Chen. This is an open-access article distributed under the terms of the Creative Commons Attribution License (CC BY). The use, distribution or reproduction in other forums is permitted, provided the original author(s) and the copyright owner(s) are credited and that the original publication in this journal is cited, in accordance with accepted academic practice. No use, distribution or reproduction is permitted which does not comply with these terms. 